\title{
Verification of Mandarin and Pummelo Somatic Hybrids by Expressed Sequence Tag-Simple Sequence Repeat Marker Analysis
}

\author{
Chunxian Chen, Jude W. Grosser ${ }^{1}$, Milica Ćalović, Patricia Serrano, Gemma Pasquali, \\ Julie Gmitter, and Fred G. Gmitter, Jr. \\ Horticultural Sciences Department, University of Florida, Institute of Food and Agricultural \\ Sciences, Citrus Research and Education Center, 700 Experiment Station Road, Lake Alfred, FL \\ 33850
}

\begin{abstract}
Additional IndeX words. Citrus aurantium, Citrus grandis, Citrus reticulata, citrus tristeza virus, CTV, protoplast fusion, sour orange rootstock

Abstract. Somatic hybridization is a powerful tool for the genetic improvement of citrus rootstocks, and it is part of an efficient in vitro-based breeding system described here. An essential component of the system is the requirement of confirming tetraploidy and the combination of the two donor genomes. Expressed sequence tag-simple sequence repeat (EST-SSR) markers provide a means to accomplish both of these objectives, and their application to a population of pummelo [Citrus grandis (L.) Osbeck] + mandarin (C. reticulata Blanco) somatic hybrids developed for the specific purpose of providing alternative rootstocks for sour orange (Citrus aurantium $\mathrm{L}$.) is detailed. Nineteen new somatic hybrids were produced from various mandarin and pummelo parents, and their ploidy level and the complementation of their nuclear genomes were confirmed using four EST-SSR markers. These markers were selected from markers previously mapped in sweet orange $[C$. sinensis $(\mathrm{L}$.) Osbeck] and trifoliate orange [Poncirus trifoliata (L.) Raf.] and prescreened for suitable allelic polymorphism within the mandarin and pummelo lines used. After polymerase chain reaction amplification of sequences from the parents and putative hybrids, the products were separated on a genetic sequencer and visualized electronically. Additionally, EST-SSR markers identified the unexpected zygotic origin of a presumed nucellar embryogenic callus line. Integration of EST-SSR techniques for high-throughput genotyping with previously developed approaches to somatic hybrid creation increases substantially the effectiveness and efficiency of this in vitro-based breeding system for citrus rootstock improvement.
\end{abstract}

Somatic hybridization is a powerful tool for the genetic improvement of citrus scion and rootstock cultivars, and the potential applications of this approach have been described previously (Grosser and Gmitter, 1990). Numerous citrus somatic hybrids have been produced since the first reports of the technique in the late 1980s. These hybrids have combined parental donors that possess complementary characteristics and have been aimed at specific genetic improvement objectives (Grosser and Gmitter, 2005; Grosser et al., 2000; Guo et al., 2004). Essentially, the methodology relies on tetraploid embryogenesis after fusion of protoplasts isolated from an embryogenic callus line with leaf mesophyll protoplasts from the complementary parent followed by the processes of validation. Somatic hybrids have been identified first by the complementary and/or intermediate morphology of regenerants followed by confirmation of ploidy level, and finally molecular markers are used to confirm the presence of alleles representing both parental diploid genomes to validate that tetraploid regenerant plants are truly combinations of two unique genomes and not simply the result of autoploidization.

Several approaches have been used to validate citrus somatic hybrids and/or their levels of ploidy. They have included mitotic chromosome counts, leaf isozyme electrophoresis,

Received for publication 13 Dec. 2007. Accepted for publication 9 Sept. 2008. This work was supported by UF-IFAS and partially funded by grants from the Florida Citrus Production Research Advisory Council and USDA-CSREES. We thank Ed Stover and James Salvatore (USDA Hort. Research Laboratory, Ft. Pierce, FL) for assistance with CTV screening and Kathy Snyder (UF-IFASCREC, Lake Alfred, FL) for manuscript preparation.

${ }^{1}$ Corresponding author. E-mail: jgrosser@ufl.edu. restriction fragment length polymorphism, genomic in situ hybridization, flow cytometry, and numerous types of molecular markers such as random amplified polymorphic DNA (RAPD), amplified fragment length polymorphism (AFLP), cleaved amplified polymorphic sequence (CAPS), and simple sequence repeat (SSR) (Deng et al., 1992; Fu et al., 2004; Grosser et al., 1995, 2004; Xu et al., 2005). The first four techniques listed are now rarely used because of their difficulty in manipulation and the low efficiencies associated with their use in acquiring the confirmatory information needed. Flow cytometry has an obvious throughput advantage over cytological methods of evaluating ploidy level; however, it does not discern donor genomes (Chen et al., 2008b; Xu et al., 2005). The latter four techniques are polymerase chain reaction (PCR)-based DNA marker systems. However, RAPDs and AFLPs, partly by virtue of their dominant nature, lack both determination locus and allelic dosage specificity and amplification reproducibility and, therefore, cannot be used reliably to determine ploidy level. The CAPS approach is very timeconsuming; it requires testing many restriction enzymes to find one able to generate cleavages of PCR amplification products revealing polymorphisms between somatic parents that can be used to confirm nuclear complementarities. SSR motifs can be found in expressed sequence tag (EST) or other genomic sequences. EST sequences represent actual genes. Increasing the number of EST sequences available in the database facilitates the development of EST-SSR markers in a batch mode (Chen et al., 2006). EST-SSR markers have a high rate of heterozygosity that allows for easy mapping and makes them useful in other applications. Citrus EST-SSRs have been used to 
characterize nucellar and zygotic progeny from diploid sour orange-like rootstock candidates (Rao et al., 2007); and more recently, selected SSRs have proven to have double capabilities, simultaneously and unambiguously determining both the sources of donor genomes and the level of ploidy in sexual hybrids (Chen et al., 2008b). In this report, we take advantage of these capabilities for validation of new citrus somatic hybrids for the first time.

A priority of Florida citrus rootstock breeding programs has been to develop replacements for sour orange rootstock that can perform similarly to sour orange but with resistance to citrus tristeza virus (CTV)-induced quick decline disease, to which it is especially sensitive (Bar-Joseph et al., 1989). Sour orange has been widely planted throughout the world because of its many positive attributes, including the ability to produce good yields of high-quality fruit that store on the tree longer than on most other citrus rootstocks. Furthermore, it is tolerant to foot rot (Phytophthora nicotianae Breda de Haan), exocortis, and xyloporosis viroids; is adapted to a wide range of soils, including high $\mathrm{pH}$ calcareous soils; and it is tolerant of citrus blight (causal organism unknown) (Castle et al., 1993; Hodgson, 1967; Saunt, 1990). Alternative rootstocks have proven to be inadequate in many cases as replacements for sour orange. For the past several years, we have been working to develop superior sour orange-like rootstock somatic hybrids by combining mandarin and pummelo parents selected for good rootstock attributes (Ananthakrishnan et al., 2006; Grosser et al., 2004, 2007b). In the present work, we describe new somatic hybrids that combine selected mandarins with superior pummelo seedlings selected from greenhouse screening tests that also showed resistance/tolerance to CTV-induced quick decline after 2 years in the field. We describe in detail the use of EST-SSR markers for the dual purposes of ploidy-level determination and confirmation of somatic hybridity as a critically useful new component to this in vitro breeding system for citrus rootstock improvement.

\section{Materials and Methods}

SElECTED PUMmelos AND MANDARINS FOR PROTOPLAST FUSION. We originally selected over 250 seedlings after germination of pummelo seed in flats of Winder soil (calcareous soil, $\mathrm{pH}=8.5$ ) inoculated with both $P$. nicotianae and Phytophthora palmivora Butler (Grosser et al., 2003). Seedlings showing good growth and nutrient uptake under these harsh conditions were selected as fusion parents and for further study (Ananthakrishnan et al., 2006; Grosser et al., 2004). These seedlings were also tested for CTV-induced quick-decline resistance by grafting each individual hybrid seedling with 'Valencia' sweet orange carrying the T-36 quick-decline isolate of CTV. Resulting 'Valencia' trees on the pummelo seedling rootstocks along with sour orange as a control were planted in a challenging field site at the University of Florida's Indian River Research and Education Center, Fort Pierce. This site featured a high $\mathrm{pH}$ calcareous Winder soil, poor drainage, and heavy sugarcane root weevil (Diaprepes abbreviatus L.) infestation. The spread of the sugarcane root weevil into most citrusgrowing regions of Florida has caused widespread tree damage, especially in the flatwoods areas, making the development of rootstocks tolerant of the diaprepes/phytophthtora complex another high-priority rootstock breeding objective (Grosser et al., 2003, 2007a). After 2 years of growth in the field, the top-performing pummelo seedlings were identified based on tree growth and overall health; and propagations of these selections were used as leaf parents in the fusion experiments presented here. Grafted plants of previously selected zygotic pummelo seedlings 'Sha Tian You' sdl-4-3-99-2, 'Siamese Sweet' sdl-7-3-99-1, 'Large Pink' sdl-7-2-99-2, and 'Hirado Buntan Pink' seedlings HBJL-3R6, HBJL3-R10, HBJL-4, HBJL-5, HBJL-7, HBJL-12, MG-1, and MG-10 were maintained in 3.8-L pots in a low-light (double shadecloth) greenhouse. Tender, fully expanded leaves from these plants were used to obtain leaf-derived protoplasts. Plants were routinely cut back to provide a continuous supply of new leaves. MG-1 and MG-10 were not included in the CTV-induced quick decline field assay and were preselected only based on soil adaptation.

Mandarin-type embryogenic suspension culture parents were chosen on the basis of performance in our protoplast culture system, general rootstock performance, wide soil adaptation, and cold-hardiness; these included 'Amblycarpa' mandarin ( $C$. amblycarpa Ochse), 'Changsha' mandarin (C. reticulata), 'Murcott' tangor and 'W. Murcott' tangor (C. reticulata $\times C$. sinensis), and 'Page' tangelo ['Minneola' tangelo $(C$. reticulata $\times C$. paradisi Macf. $) \times$ 'Clementine' mandarin (C. reticulata)]. Embryogenic suspension cultures of 'Amblycarpa' mandarin, 'Changsha' mandarin, 'Page' tangelo, 'Murcott' tangor, and 'W. Murcott' tangor were initiated from unfertilized ovule-derived friable embryogenic callus cultures maintained in the citrus embryogenic callus collection of the University of Florida's Citrus Research and Education Center, Lake Alfred (CREC) (Grosser and Gmitter, 1990). All callus lines were initiated at CREC with the exception of the 'Page' tangelo line (provided by W.W. Guo). Suspension-derived protoplasts were isolated from 6-month- to 2-year-old suspension cultures maintained in $\mathrm{H}+\mathrm{H}$ medium on a 2 -week subculture cycle with protoplasts isolated during Days 4 through 12 (Grosser and Gmitter, 1990).

Protoplast isolation and fusion. Protoplasts were isolated from the 'Amblycarpa' mandarin, 'Changsha' mandarin, 'Page' tangelo, 'Murcott' tangor, and 'W. Murcott' tangor suspension cultures in a 2.5:1.5 (v:v) mixture of $0.7 \mathrm{M} \mathrm{BH}$ protoplast culture medium and enzyme solution according to Grosser and Gmitter (1990). Before protoplast isolation, selected leaves from greenhouse parental plants were surfacesterilized by immersion in $1 \mathrm{~N} \mathrm{HCl}$ for $5 \mathrm{~s}$ followed by immersion in $20 \%$ commercial bleach for $15 \mathrm{~min}$ and rinsed three times with sterile distilled water for $10 \mathrm{~min}$. Sterile leaves were feather-cut with a sharp scalpel and incubated overnight (including a 25-min vacuum infiltration) in a 8:3 (v:v) mixture of $0.6 \mathrm{M} \mathrm{BH} 3$ protoplast culture medium and modified enzyme solution (Grosser and Gmitter, 1990). Modification of the enzyme solution included the elimination of Pectolyase Y-23 and the doubling of Onozuka RS cellulose and Macerozyme R-10 from $1 \%$ to $2 \%$. This modification was necessary because commercial suppliers of Pectolyase Y-23 changed fungal sources, and the new product no longer facilitated citrus protoplast isolation. Protoplasts from both suspension and leaf sources were purified by passage through a $45-\mu \mathrm{m}$ stainless steel mesh screen and then by centrifugation on $25 \%$ sucrose and 13\% mannitol gradient (Grosser and Gmitter, 1990).

Our highly successful method of fusing embryogenic culture-derived protoplasts of one parent with leaf-derived protoplasts of the second parent was used in all experiments (Grosser 
and Gmitter, 1990; Grosser et al., 2000). Fusions were conducted in $60 \times 15-\mathrm{mm}$ polystyrene petri dishes using our standard PEG (40\% polyethylene glycol) volumetric plating method (Grosser and Gmitter, 1990). After fusion, protoplasts were cultured in a $1: 1(\mathrm{v}: \mathrm{v})$ mixture of $0.6 \mathrm{M} \mathrm{BH} 3$ and $0.6 \mathrm{M}$ EME protoplast culture media (Grosser and Gmitter, 1990), and petri dishes sealed with Nescofilm (Karlan, Cottonwood, AZ) were maintained in plastic boxes under low light in a tissue culture room.

Plant Recovery and ploidy analysis. Regenerating embryogenic callus was transferred to solid EME medium containing $50 \mathrm{~g} \cdot \mathrm{L}^{-1}$ maltose (Perez et al., 1998) for somatic embryo induction according to Grosser and Gmitter (1990). Regenerated embryos were cultured over $0.22-\mu \mathrm{m}$ cellulose acetate membrane filters placed on fresh plates of EME-maltose solid medium to normalize and enlarge the embryos (Niedz et al., 2002). After 4 weeks, embryos were cultured for one passage on 1500 embryo enlargement medium (Grosser and Gmitter, 1990). Large somatic embryos, often exhibiting abnormal shapes, were screened for ploidy level using a tabletop flow cytometer (model D-48161; Partec, Münster, Germany), as previously described (Kahn and Grosser, 2004). All confirmed tetraploid embryos were sliced horizontally with a sharp scalpel and transferred directly to DBA3 medium for adventitious shoot induction and multiplication (Deng et al., 1992). Diploid embryos were discarded, except for those regenerating from fusions involving ' $\mathrm{W}$. Murcott' tangor, which were kept for a somaclonal variation experiment. Developing tetraploid shoots were rooted on RMAN medium in Magenta boxes (Grosser and Gmitter, 1990). Plantlets were transplanted into Cone-tainers (Stuewe \& Sons, Corvallis, OR) filled with commercial potting mix, covered with clear rigid plastic for 2 to 4 weeks for acclimation, and maintained in a heavily shaded greenhouse for 2 to 3 months.

Simple SEQUENCE REPEAT ANALYSIS FOR SOMATIC HYBRID VERIFICATION. Genomic DNA samples were prepared from young leaves of all donor parents and putative somatic hybrids using a GenElute Plant Genomic DNA miniprep kit (Sigma, St. Louis, MO). In addition, a DNA sample was also extracted from the embryogenic suspension line of 'Page' tangelo for comparisons with that from the leaves of a true-to-type 'Page' tangelo tree; this was done because results from putative 'Page' tangelo-derived somatic hybrids possessed alleles different from what would be seen if 'Page' tangelo was the donor parent. Four EST-SSR primer sets designated CX0010, CX0035, CX2007, and CX2021 were synthesized by Operon Technologies (Huntsville, AL) and were used to amplify DNA sequences from all parents and derived putative somatic hybrids for genotyping. These four were chosen because they were previously found to be heterozygous in sweet orange and trifoliate orange, each with two different alleles (Chen et al., 2008a) as well as in some mandarin and pummelo (C. Chen, unpublished data), and they had been mapped in both sweet orange and trifoliate orange genomes (Chen et al., 2008a). The primer labeling, PCR program, and preparation of PCR products to load onto the Genetic Analyzer 3130xl (Applied Biosystems, Foster City, CA) were previously described in detail (Chen et al., 2006). The generated ABI proprietary files with the chromatographic trace data were analyzed using GeneMarker (SoftGenetics, State College, PA) to produce an allele table.

\section{Results and Discussion}

VERIFICATION OF SOMATIC HYBRIDS BY EXPRESSED SEQUENCE TAG-SIMPLE SEQUENCE REPEAT MARKER ANALYSIS. Selected ESTSSRs have proven to have double capabilities, simultaneously and unambiguously determining both the sources of donor genomes and the level of ploidy in sexual hybrids (Chen et al., 2008b). Such application can be directly extended to quick verification of somatic hybrids that are produced by simple addition of two genomes. According to the number of alleles and amplified DNA fragments, a codominant EST-SSR marker in a diploid individual can have two homozygous alleles that are the same or two heterozygous alleles that are different. When such loci in diploids are genotyped and visualized using an ABI Genetic Analyzer, the former will be detected as only one peak (representing the amplified DNA fragment), and the latter will result in two different peaks. For example, as shown in Figure 1, CX0010 is homozygous (two identical alleles producing one peak) in 'Changsha' mandarin, heterozygous (two different alleles producing two peaks) in HBJL-5 pummelo, and combined in their somatic hybrid 'Changsha' + HBJL-5. Therefore, somatic hybrids can be verified as the alleles detected at any locus are combined from the two donor parents.

A detailed allele (peak) table was generated using the four selected marker loci, CX0010, CX0035, CX2007, and CX2021, in all parents and putative somatic hybrids (Table 1). After sorting any two parents and their putative somatic hybrids together, we found all somatic hybrids, except those from 'Page' tangelo, had the alleles directly combined from their two donor parents. The 'Page' tangelo tree, from which the leaf DNA was extracted and genotyped, apparently was not the donor parent of the somatic hybrids because unexpected alleles were detected at the four loci when compared with the genotyping results from its embryonic suspension line DNA sample (Table 2), which was the true donor genotype used in Table 1 .

In general, SSR markers can be used to confirm somatic hybrids as long as there is polymorphism between the two donor parents. All confirmative markers for these somatic combinations are summarized in Table 3 and are marked with bold alleles in Table 1. The ideal SSR markers are those that

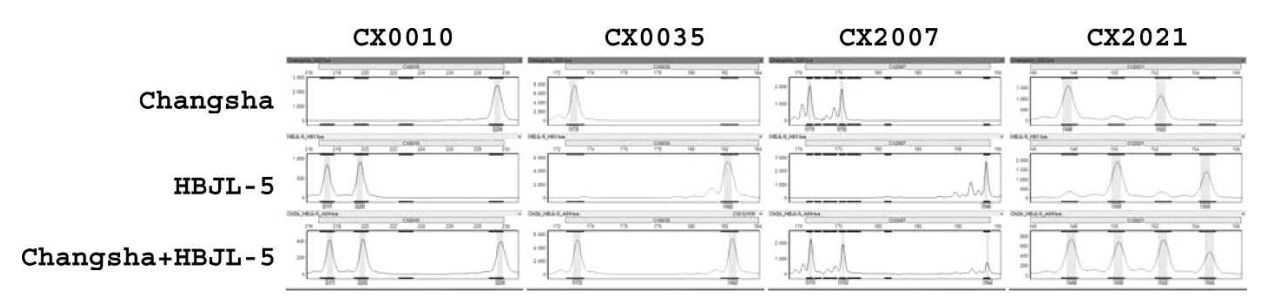

Fig. 1. A graphic example of expressed sequence tag-simple sequence repeat (EST-SSR) genotypes in two donor parents and their putative hybrid. It was generated from ABI trace files by GeneMarker (SoftGenetics, State College, PA). Four SSR primers (loci), CX0010, CX0035, CX2007, and CX2021, were used. The somatic hybrid, 'Changsha' mandarin + HBJL-5 pummelo, was verified as its alleles (indicated as positional peaks in this figure and sizes in Table 1) at each locus were a combination of the alleles unique to both donor parents, 'Changsha' and HBJL-5. 
Table 1. Detected alleles [represented by different-sized amplified fragments measured in basepairs (bps)] at four markers in mandarins and pummelos and their corresponding somatic hybrids.

\begin{tabular}{|c|c|c|c|c|c|c|c|c|c|c|c|c|c|}
\hline \multirow{4}{*}{$\frac{\text { Mandarin parents }^{\mathrm{z}}}{\text { Amblycarpa mandarin }}$} & \multirow{4}{*}{$\begin{array}{l}\text { Pummelo parents } \\
\text { and somatic hybrids }\end{array}$} & \multicolumn{12}{|c|}{ EST-SSR primer sets (markers) } \\
\hline & & \multicolumn{3}{|c|}{ CX0010 } & \multicolumn{2}{|c|}{ CX0035 } & \multicolumn{3}{|c|}{ CX2007 } & \multicolumn{4}{|c|}{ CX2021 } \\
\hline & & \multicolumn{12}{|c|}{ Detected alleles [amplified fragments (bp)] } \\
\hline & & $\underline{220}$ & & & $\underline{173}$ & $\underline{182}$ & $\underline{177}$ & & & $\underline{148}$ & $\underline{150}$ & & \\
\hline & $4-3-99-2$ & 217 & & & 182 & & 174 & 181 & & 159 & & & \\
\hline & Amblycarpa + 4-3-99-2 & 217 & 220 & & 173 & 182 & 174 & 177 & 181 & 148 & 150 & 159 & \\
\hline & 7-3-99-1 & 220 & & & 182 & & 179 & & & 150 & 159 & & \\
\hline & Amblycarpa + 7-3-99-1 & 220 & & & 173 & 182 & 177 & 179 & & 148 & 150 & 159 & \\
\hline & HBJL-3R10 & 217 & & & 182 & & 194 & & & 142 & 150 & & \\
\hline & Amblycarpa + HBJL-3R10 & 217 & 220 & & 173 & 182 & 177 & 194 & & 142 & 148 & 150 & \\
\hline & HBJL-5 & 217 & 220 & & 182 & & 194 & & & 150 & 155 & & \\
\hline & Amblycarpa + HBJL-5 & 217 & 220 & & 173 & 182 & 177 & 194 & & 148 & 150 & 155 & \\
\hline & HBJL-7 & 217 & 220 & & 182 & & 194 & & & 150 & 155 & & \\
\hline & Amblycarpa + HBJL-7 & 217 & 220 & & 173 & 182 & 177 & 194 & & 148 & 150 & 155 & \\
\hline & MG-1 & 217 & 220 & & 182 & & 194 & & & 150 & 152 & & \\
\hline & Amblycarpa + MG-1 & 217 & 220 & & 173 & 182 & 177 & 194 & & 148 & 150 & & \\
\hline & MG-10 & 217 & 220 & & 182 & & 194 & & & 150 & & & \\
\hline & Amblycarpa + MG-10 & 217 & 220 & & 173 & 182 & 177 & 194 & & 148 & 150 & & \\
\hline Changsha mandarin & & $\underline{229}$ & & & $\underline{173}$ & & $\underline{171}$ & $\underline{175}$ & & $\underline{148}$ & $\underline{152}$ & & \\
\hline & HBJL-3R6 & 217 & 220 & & 182 & & 194 & & & 142 & 150 & & \\
\hline & Changsha + HBJL-3R6 & 217 & 220 & 229 & 173 & 182 & 171 & 175 & 194 & 142 & 148 & 150 & 155 \\
\hline & HBJL-5 & 217 & 220 & & 182 & & 194 & & & 150 & 155 & & \\
\hline & Changsha + HBJL-5 & 217 & 220 & 229 & 173 & 182 & 171 & 175 & 194 & 148 & 150 & 152 & 155 \\
\hline & HBJL-7 & 217 & 220 & & 182 & & 194 & & & 150 & 155 & & \\
\hline & Changsha + HBJL-7 & 217 & 220 & 229 & 173 & 182 & 171 & 175 & 194 & 148 & 150 & 152 & 155 \\
\hline Page $^{x}$ tangelo & & $\underline{229}$ & & & $\underline{182}$ & & $\underline{171}$ & $\underline{177}$ & & $\underline{148}$ & $\underline{150}$ & & \\
\hline & HBJL-3R10 & 217 & & & 182 & & 194 & & & 142 & 150 & & \\
\hline & Page + HBJL-3 & 217 & 229 & & 182 & & 171 & 177 & 194 & 142 & 148 & 150 & \\
\hline & HBJL-4 & 217 & & & 182 & & 194 & & & 150 & 155 & & \\
\hline & Page + HBJL-4 & 217 & 229 & & 182 & & 171 & 177 & 194 & 148 & 150 & 155 & \\
\hline & HBJL-5 & 217 & 220 & & 182 & & 194 & & & 150 & 155 & & \\
\hline & Page + HBJL-5 & 217 & 220 & 229 & 182 & & 171 & 177 & 194 & 148 & 150 & 155 & \\
\hline & HBJL-7 & 217 & 220 & & 182 & & 194 & & & 150 & 155 & & \\
\hline & Page + HBJL-7 & 217 & 220 & 229 & 182 & & 171 & 177 & 194 & 148 & 150 & 155 & \\
\hline & HBJL-12 & 217 & & & 182 & & 194 & & & 150 & 155 & & \\
\hline & Page + HBJL-12 & 217 & 229 & & 182 & & 171 & 177 & 194 & 148 & 150 & 155 & \\
\hline Murcott tangor & & $\underline{229}$ & & & $\underline{182}$ & & $\underline{171}$ & $\underline{177}$ & & $\underline{150}$ & $\underline{155}$ & & \\
\hline & HBJL-5 & 217 & 220 & & 182 & & 194 & & & 150 & 155 & & \\
\hline & Murcott + HBJL-5 & 217 & 220 & 229 & 182 & & 171 & 177 & 194 & 150 & 155 & & \\
\hline W. Murcott tangor & & $\underline{220}$ & $\underline{229}$ & & $\underline{173}$ & $\underline{182}$ & $\underline{177}$ & & & $\underline{150}$ & $\underline{152}$ & & \\
\hline & $7-2-99-2$ & 217 & & & 182 & & 194 & & & 150 & $\overline{152}$ & & \\
\hline & W. Murcott + 7-2-99-2 & 217 & 220 & 229 & 173 & 182 & 177 & 194 & & 150 & 152 & & \\
\hline & HBJL-3R6 & 217 & 220 & & 182 & & 194 & & & 150 & 155 & & \\
\hline & W. Murcott + HBJL-3R6 & 217 & 220 & 229 & 173 & 182 & 177 & 194 & & 150 & 152 & 155 & \\
\hline & HBJL-7 & 217 & 220 & & 182 & & 194 & & & 150 & 155 & & \\
\hline & W. Murcott + HBJL-7 & 217 & 220 & 229 & 173 & 182 & 177 & 194 & & 150 & 152 & 155 & \\
\hline
\end{tabular}

${ }^{\mathrm{z}}$ Each mandarin parent, with alleles underlined and italic, was listed once at the top and followed by every combined pummelo parent and their somatic hybrid. yThe bold alleles, which were derived from a combination of those distinct in two donor parents, indicated that the somatic hybrids were unambiguously confirmed by the markers.

${ }^{x}$ The 'Page' tangelo genotype listed here was generated by the genomic DNA from the embryogenic suspension line of 'Page' tangelo. It was different from that in the leaf of the cultivar (Table 2).

EST-SSR = expressed sequence tag-simple sequence repeat. 
Table 2. Detected alleles [represented by different-sized amplified fragments measured in basepairs (bps)] in 'Page' tangelo suspension and leaf DNA samples at four markers.

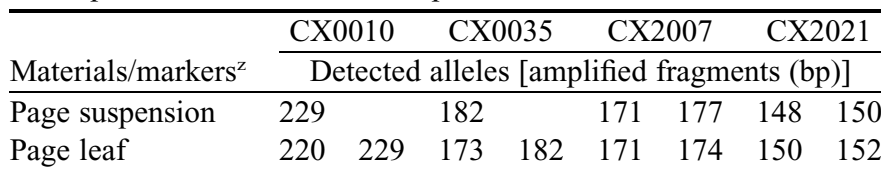

"'Page' suspension = DNA extracted from the embryogenic suspension line of 'Page' tangelo, 'Page' leaf = DNA extracted from the leaf of the true-to-type 'Page' tangelo. Compared with 'Page' leaf, new homozygosity at CX0010 and CX0035 and new alleles at CX2007 and CX2021 in 'Page' suspension indicated the embryonic suspension line was of zygotic origin.

have four distinct heterozygous alleles in two donor parents (Chen et al., 2008b). In other words, at one locus, Parent 1 has allele a and b, 2 has $\mathrm{c}$ and $\mathrm{d}$, and their somatic hybrids between 1 and 2 would detect a, b, c, and d at the locus, simultaneously indicating the allele parental source and the tetraploid condition of the hybrid. The marker CX2021 in the combination of 'Changsha' mandarin and HBJL-5 pummelo was such a case. Two heterozygous alleles, 148 and 152, were detected in 'Changsha' mandarin, 150 and 155 in HBJL-5, and all four alleles, $148,152,150$, and 155 , as a result of somatic addition, were observed in their somatic hybrid, 'Changsha' + HBJL-5 (Table 1; Fig. 1).

EXPRESSED SEQUENCE TAG-SIMPLE SEQUENCE REPEAT MARKER ANALYSIS REVEALS THE ZYGOTIC ORIGIN OF THE 'PAGE' TANGELO EMBRYOGENIC SUSPENSION LINE. SSR analysis of the 'Page' tangelo embryogenic suspension line surprisingly revealed that it was not true-to-type 'Page' tangelo of nucellar origin, although 'Page' tangelo generally produces developed seeds that contain nucellar embryos. The genotypes from the suspension line at any of the EST-SSR loci surveyed, not those from the true-to-type 'Page' tangelo tree leaf, matched the expected addition of the alleles in all the somatic hybrids from any 'Page' tangelo and pummelo combination. All four marker loci exhibited differences between the DNA samples from the suspension line and tree leaf of 'Page' tangelo (Table 2). CX2021 and CX2007 exhibited only one 'Page' tangelo allele along with a new foreign allele, suggesting that the callus line was of zygotic origin from crosspollination and not from selfing. The embryogenic 'Page' tangelo callus line used to initiate the suspension culture was initiated from an undeveloped and presumably unfertilized ovule, but it may have contained a small zygotic embryo, resulting in callus proliferation from the zygote rather than from the nucellar tissues (W.W. Guo, personal communication). Alternatively, there may have been a labeling error during embryogenic callus or suspension transfer, and this possibility is being investigated. This unexpected finding suggests that the identity of all embryogenic callus lines should be validated by SSR analysis. Somatic hybrids produced using this putative zygotic 'Page' tangelo callus line, therefore, contain genomic contributions of unidentified origin.

Parent Combinations and NuRsery performance of PUTATIVE SOMATIC HYBRIDS. Somatic hybrid plants were regenerated from 19 new mandarin + pummelo parental combinations (Table 3). All regenerated allotetraploid somatic hybrid plants produced in this study exhibited leaf morphologies intermediate to that of their parents, and all but one of the new hybrids showed good nursery performance (Table 3); they were similar to sour orange in appearance and nursery performance. After somatic fusion, somatic embryo induction and plant recovery were highly efficient for all fusions involving 'Amblycarpa' mandarin and ' $\mathrm{W}$. Murcott' tangor as the embryogenic suspension parents. Large numbers of diploid embryos were recovered from fusions involving these two suspension parents, and ploidy analysis through flow cytometry proved to be an effective screening tool to separate out the tetraploids early on, thereby saving time and resources. Large numbers of tetraploid somatic hybrid plants (generally greater

Table 3. Nursery vigor and confirmative EST-SSR markers of mandarin and pummelo somatic hybrids. ${ }^{\mathrm{z}}$

\begin{tabular}{|c|c|c|c|c|c|}
\hline & \multicolumn{5}{|c|}{ Mandarin parent } \\
\hline & Amblycarpa mandarin & Changsha mandarin & Page tangelo & Murcott tangor & W. Murcott tan \\
\hline & \multicolumn{5}{|c|}{ Somatic hybrid nursery vigor and confirmative EST-SSR markers } \\
\hline Pummelo parent & & & & & \\
\hline $4-3-99-2$ & $\mathrm{H}^{\mathrm{y}}\left(1,3,4^{\mathrm{x}}\right)$ & & & & \\
\hline $7-2-99-2$ & & & & & $\mathrm{H}(1,3)$ \\
\hline $7-3-99-1$ & $\mathrm{H}(3,4)$ & & & & \\
\hline HBJL-3R6 & & $\mathrm{M}(1,2,3,4)$ & & & $\mathrm{H}(1,3,4)$ \\
\hline HBJL-3R 10 & $\mathrm{H}(1,3,4)$ & & $\mathrm{M}(1,3,4)$ & & \\
\hline HBJL-4 & & & $M(1,3,4)$ & & \\
\hline HBJL-5 & $H(3,4)$ & $\mathrm{H}(1,2,3,4)$ & $\mathrm{H}(1,3,4)$ & $\mathrm{L}(1,3)$ & $\mathrm{H}(1,3,4)$ \\
\hline HBJL-7 & $\mathrm{H}(3,4)$ & $\mathrm{H}(1,2,3,4)$ & $M(1,3,4)$ & & \\
\hline HBJL-12 & & & $\mathrm{H}(1,3,4)$ & & \\
\hline MG-1 & $\mathrm{H}(3,4)$ & & & & \\
\hline MG-10 & $\mathrm{H}(3)$ & & & & \\
\hline
\end{tabular}

$\overline{{ }^{\mathrm{z}} \text { Five mandarins (in column) and } 11 \text { seedling pummelos (in row) were used for somatic hybridization parents. However, only combinations of }}$ mandarin and pummelo where the data are filled were selected or successful to produce new allotetraploid somatic hybrids plants.

y The letters, H, M, and L, indicated the comparable nursery vigor of these somatic hybrids. $\mathrm{H}=$ high, comparable to sour orange; $\mathrm{M}=$ medium, comparable to Swingle citrumelo [Citrus paradisi Macf. $\times$ Poncirus trifoliata (L.) Raf.]; L $=$ low, comparable to Flying Dragon [Poncirus trifoliata (L.) Raf.].

${ }^{x}$ The numbers 1, 2,3, and 4 in the parentheses represented which EST-SSR markers of the four that were able to confirm the somatic hybrids (with bold alleles in Table 1). $1=$ CX0100, $2=$ CX0035, $3=$ CX2007, and $4=$ CX2021.

EST-SSR $=$ expressed sequence tag-simple sequence repeat. 
than 50 per combination) were recovered from fusions involving these two embryogenic parents (data not shown). Some diploid plants were intentionally regenerated from fusions involving ' $\mathrm{W}$. Murcott' tangor to create a population of protoclones for subsequent study of somaclonal variation in this promising new scion cultivar, also known as 'Nadorcott' (a chance hybrid of 'Murcott' tangor $\times$ an unknown) and 'Afourer' (Nadori, 2006). 'W. Murcott' tangor was chosen as a rootstock parent in this study because its seed are highly nucellar, produce vigorous uniform seedlings in the nursery (unpublished data), and because of its good performance in protoplast isolation and culture. 'W. Murcott' tangor is also being used as a scion fusion parent with good success (unpublished data). 'Amblycarpa' mandarin was chosen as a fusion parent as a result of its outstanding previous performance in our rootstock somatic hybridization program (Ananthakrishnan et al., 2006; Grosser et al., 2004, 2007b) and good performance in rootstock trials on challenging soils with high clay content and porous-limestone soils (Castle et al., 2004; Medina-Urrutia, 1996). 'Murcott' tangor is a seedy polyembryonic tangor that has only occasionally been included in rootstock trials in Florida with promising results regarding tree growth, yield, and fruit quality (W.S. Castle, personal communication). 'Changsha' mandarin was chosen as a fusion parent because it has been previously shown to be a good parent in sexual hybridization for citrus rootstock improvement (Pompei et al., 2002), and it has exceptional cold-hardiness among mandarin types (Sauls and Jackson, 2000). 'Page' tangelo was chosen as a parent because of its previous good performance in protoplast isolation and culture (Guo et al., 2004). Somatic embryo induction and plant recovery from fusions involving 'Changsha' mandarin, 'Murcott' tangor, and 'Page' tangelo as the embryogenic parents were much less efficient than those from 'W. Murcott' tangor and 'Amblycarpa' mandarin with only a few abnormal embryos recovered from each parental combination. Somatic hybrid plant recovery from these combinations required adventitious shoot induction from enlarged embryos sliced in half and cultured on DBA3 shoot induction medium (Deng et al., 1992). Only small numbers of somatic hybrid plants (less than 10) were recovered from these combinations. In the case of fusions involving 'Murcott' tangor, only tetraploid embryos were recovered, because this suspension line lost its ability to regenerate plants directly, possibly as a result of the accumulation of cytological mutations over time (Grosser et al., 2000). Such mutations could also contribute to the lack of vigor in the regenerated somatic hybrid 'Murcott' tangor + HBJL-5 pummelo (Table 3).

Propagated trees have been entered into various tests to determine horticultural performance and tolerance/resistance to CTV-induced quick decline, citrus blight, and the diaprepes/ phytophthora complex. Seed trees of each hybrid have also been produced and planted in the field to subsequently determine the amenability of each hybrid to standard nursery propagation through nucellar seed production. EST-SSR techniques will be useful to determine whether the seedling offspring of these hybrids are nucellar or zygotic.

In conclusion, the unambiguous identification and confirmation of tetraploid somatic hybrids is facilitated by the application of EST-SSR marker genotyping using genetic sequence technology. Both ploidy level and allelic complementarities were determined simultaneously by appropriate selection of EST-SSR marker loci in the parental lines. This technology also very clearly identified the zygotic origin of a presumed nucellar callus line. Combining EST-SSR markers along with flow cytometry analysis of developing embryos greatly enhances the efficiency of citrus somatic hybridization as a tool for the genetic improvement of rootstocks. As illustrated in this case, 19 new somatic hybrids (15 combining mandarin types with CTV-tolerant/-resistant pummelos) were generated in efforts to develop CTV-induced quick declineresistant replacement rootstocks for sour orange.

A key feature of this in vitro rootstock breeding system is that it provides an opportunity to preserve superior diploid genomes in allotetraploid somatic hybrids. Citrus rootstock improvement is a continuum and like with all new rootstock hybrids, several years of field testing will be required to determine if horticultural performance, fruit-holding capacity, soil adaptation, and survivability will be adequate for any of the new somatic hybrid rootstock candidates reported here to be released as improved commercial rootstocks. Hybrids that perform well but do not produce adequate nucellar seeds for traditional nursery propagation may be used as female parents in our tetraploid rootstock breeding program.

\section{Literature Cited}

Ananthakrishnan, G., M. Calovic, P. Serrano, and J.W. Grosser. 2006. Production of additional allotetraploid somatic hybrids combining mandarins and sweet orange with pre-selected pummelos as potential candidates to replace sour orange rootstock. In Vitro Cell. Dev. Biol. Plant 42:367-371.

Bar-Joseph, M., R. Marcus, and R.F. Lee. 1989. The continuous challenge of citrus tristeza virus control. Annu. Rev. Phytopathol. 27:291-316.

Castle, W.S., J.W. Grosser, F.G. Gmitter, Jr., R.J. Schell, T. AyalaSilva, J.H. Crane, and K.D. Bowman. 2004. Evaluation of new citrus rootstocks for 'Tahiti' lime production in southern Florida. Proc. Florida State Hort. Soc. 117:174-181.

Castle, W.S., D.P.H. Tucker, A.H. Krezdorn, and C.O. Youtsey. 1993. Rootstocks for Florida citrus. 2nd Ed. Univ. Florida Publ. SP-42.

Chen, C., K.D. Bowman, Y.A. Choi, P.M. Dang, M.N. Rao, S. Huang, J.R. Soneji, T.G. McCollum, and F.G. Gmitter, Jr. 2008a. EST-SSR genetic maps for Citrus sinensis and Poncirus trifoliata. Tree Genet. Genomes 4:1-10.

Chen, C., M.T. Lyon, D. O’Malley, C.T. Federici, J. Gmitter, J.W. Grosser, J.X. Chaparro, M.L. Roose, and F.G. Gmitter, Jr. 2008b. Origin and frequency of $2 \mathrm{n}$ gametes in Citrus sinensis $\times$ Poncirus trifoliata and their reciprocal crosses. Plant Sci. 174:1-8.

Chen, C., P. Zhou, Y.A. Choi, S. Huang, and F.G. Gmitter, Jr. 2006. Mining and characterizing microsatellites from citrus ESTs. Theor. Appl. Genet. 112:1248-1257.

Deng, X.X., J.W. Grosser, and F.G. Gmitter, Jr. 1992. Intergeneric somatic hybrid plants from protoplast fusion of Fortunella crassifolia cultivar 'Meiwa' with Citrus sinensis cultivar 'Valencia'. Scientia Hort. 49:55-62.

Fu, C.H., C.L. Chen, W.W. Guo, and X.X. Deng. 2004. GISH, AFLP, and PCR-RFLP analysis of an intergeneric somatic hybrid combining Goutou sour orange and Poncirus trifoliata. Plant Cell Rpt. 23:391396.

Grosser, J.W. and F.G. Gmitter, Jr. 1990. Protoplast fusion and citrus improvement. Plant Breed. Rev. 8:339-374.

Grosser, J.W. and F.G. Gmitter, Jr. 2005. Thinking outside the cell-Applications of somatic hybridization and cybridization in crop improvement, with citrus as a model. In Vitro Cell. Dev. Plant 41:220-225.

Grosser, J.W., F.G. Gmitter, Jr., and W.S. Castle. 1995. Production and evaluation of citrus somatic hybrid rootstocks: Progress report. Proc. Florida State Hort. Soc. 108:140-143. 
Grosser, J.W., J.H. Graham, A. Hoyte, H.M. Rubio, D.B. Bright, J. Gmitter, C.X. Chen, and F.G. Gmitter, Jr. 2007a. Continued development of rootstocks tolerant of the diaprepes/phytophthora complex. Proc. Florida State Hort. Soc. 120:103-109.

Grosser, J.W., J.L. Chandler, and L.W. Duncan. 2007b. Production of mandarin + pummelo somatic hybrid citrus rootstocks with potential for improved tolerance/resistance to sting nematode. Scientia Hort. 113:33-36.

Grosser, J.W., J.H. Graham, C.W. McCoy, A. Hoyte, H.M. Rubio, D.B. Bright, and J.L. Chandler. 2003. Development of 'Tetrazyg' rootstocks tolerant of the diaprepes/phytophthora complex under greenhouse conditions. Proc. Florida State Hort. Soc. 116:262-267.

Grosser, J.W., P. Ollitrault, and O. Olivares-Fuster. 2000. Somatic hybridization in citrus: An effective tool to facilitate variety improvement. In Vitro Cell. Dev. Biol. Plant 36:434-449.

Grosser, J.W., V.M. Urrutia, G. Ananthakrishnan, and P. Serrano. 2004. Building a replacement sour orange rootstock: Somatic hybridization of selected mandarin + pummelo combinations. J. Amer. Soc. Hort. Sci. 129:530-534.

Guo, W.W., D. Prasad, P. Serrano, F.G. Gmitter, Jr., and J.W. Grosser. 2004. Citrus somatic hybridization with potential for direct tetraploid cultivar development. J. Hort. Sci. Biotechnol. 79:400-405.

Hodgson, R.W. 1967. Horticultural varieties of citrus, p. 431-591. In: Reuther, W., H.J. Webber, and L.D. Batchelor (eds.). The citrus industry. Univ. California, Div. Agr. Sci., Riverside, CA.

Kahn, I.A. and J.W. Grosser. 2004. Regeneration and characterization of somatic hybrid plants of Citrus sinensis (sweet orange) and Citrus micrantha, a progenitor species of lime. Euphytica 137:271-278.
Medina-Urrutia, V.M. 1996. Comportamiento de portainjertos de limón mexicano en Colima. Instituto Nacional de Investigaciones Forestales y Agropecuarias (Mexico) - Centro de Investigación Regional del Pacífico Centro-Campo Experimental Tecoman. Folleto Tecnico No. 3.

Nadori, E.B. 2006. Nadorcott mandarin: A promising new variety. Proc. Intl. Soc. Citriculture 1:356-359.

Niedz, R.P., S.E. Hyndman, E.T. Wynn, and M.G. Bausher. 2002. Normalizing sweet orange [C. sinensis (L.) Osbeck] somatic embryogenesis with semi-permeable membranes. In Vitro Cell. Dev. Biol. Plant 38:552-557.

Perez, R.M., A.M. Galiana, L. Navarro, and N. Duran-Vila. 1998. Embryogenesis in vitro of several Citrus species and cultivars. J. Hort. Sci. Biotechnol. 73:796-802.

Pompei, J., Jr., F.F. Laranjeira, and S. Blumer. 2002. 'Valencia' sweet orange trees grafted on trifoliate hybrids. Sci. Agr. (Piracicaba, Brazil) 59:93-97.

Rao, M.N., J.R. Soneji, C. Chen, S. Huang, and F.G. Gmitter, Jr. 2007. Characterization of zygotic and nucellar seedlings from sour orangelike citrus rootstock candidates using RAPD and EST-SSR markers. Tree Genet. Genomes 4:113-124.

Sauls, J.W. and L.K. Jackson. 2000. Cold-hardy citrus for north Florida. Univ. Florida Coop. Ext. Serv. FC-36.

Saunt, J. 1990. Citrus varieties of the world: An illustrated guide. Sinclair Intl., Norwich, UK.

Xu, X.Y., J.H. Liu, and X.X. Deng. 2005. FCM, SSR, and CAPS analysis of intergeneric somatic hybrid plants between Changshou kumquat and Dancy tangerine. Bot. Bul. Acad. Sinica 46:93-98. 